\title{
A Model of a Marine Two-Stroke Diesel Engine with EGR for Low Load Simulation
}

\author{
Xavier Llamas Lars Eriksson \\ Vehicular Systems, Dept. of Electrical Engineering, Linköping University, Sweden \\ \{xavier.llamas. comellas, lars.eriksson\} eliu.se
}

\begin{abstract}
A mean value engine model of a two-stroke marine diesel engine with EGR that is capable of simulating during low load operation is developed. In order to be able to perform low load simulations, a compressor model capable of low speed extrapolation is also investigated and parameterized for two different compressors. Moreover, a parameterization procedure to get good parameters for both stationary and dynamic simulations is described and applied. The model is validated for two engine layouts of the same test engine but with different turbocharger units. The simulation results show a good agreement with the different measured signals, including the oxygen content in the scavenging manifold.
\end{abstract}

Keywords: modeling, parameterization, simulations, exhaust gas recirculation, combustion engines

\section{Introduction}

The marine shipping industry is facing increased demands in the reduction of harmful exhaust gas emissions. Stricter emission limits of Sulphur Oxides (SOx) and Nitrogen Oxides (NOx) are imposed in certain Emission Control Areas (ECAs). The emission values to fulfill in these ECAs are set by the IMO Tier III limits (International Maritime Organization, 2013) that came into play in January 2016. One of the available technical solutions to achieve the targeted reduction in NOx emissions is Exhaust Gas Recirculation (EGR). An EGR system recirculates a fraction of the exhaust gas into the scavenging manifold, providing burned gases in the combustion chamber that directly decreases the production of NOx during the combustion.

EGR technologies for two-stroke engines are still at the initial phases of its development. In addition, there are not many available vessels with an EGR system installed and thus performing tests is often difficult. Furthermore, testing any new system in marine two-stroke engines is also very costly mainly due to the fuel cost associated with the sizes of such engines. Hence, in order to improve the performance of the EGR control systems, a fast and accurate simulation model is a very valuable tool.

Mean Value Engine Models (MVEMs), are a very common approach for control oriented modeling of internal combustion engines. In particular, EGR systems have been also modeled using this approach. Many interesting research articles about EGR modeling in automotive applications can be found in the literature, some examples are, (Wahlström and Eriksson, 2011; Nieuwstadt et al., 2000). On the other hand, marine two-stroke engines have not been widely studied. Nevertheless, some research papers focused on MVEMs for two-stroke engines are (Blanke and Anderson, 1985; Theotokatos, 2010; Hansen et al., 2013). In addition, in (Guan et al., 2014) the modeling of the low load operation of a two-stroke engine without EGR is studied.

The work presented here is an extension of the model proposed in (Alegret et al., 2015), which enables the model to simulate low engine loads. The low load operation is very relevant for the EGR control since the Tier III emission limits have to be fulfilled near certain coasts, e.g. harbors, where the vessel is normally operating at low loads. The main new component that needs to be introduced for this low load simulation is the auxiliary electrical blower. Its mission is to ensure that there is enough scavenging pressure at low loads when the turbocharger is not capable to provide it. Moreover, the turbocharger model will be required to simulate at low speeds and pressure ratios. This area is normally not measured in the provided performance maps, so a model that can extrapolate to this area is also required.

The developed model is, as in (Alegret et al., 2015), based on the 4T50ME-X test engine from MAN Diesel \& Turbo. It is a two-stroke uniflow diesel engine, turbocharged, with variable valve timing and direct injection. Its maximum rated power is $7080 \mathrm{~kW}$ at $123 \mathrm{rpm}$. Also, it is equipped with an EGR system and a Cylinder Bypass Valve (CBV).

\section{Experimental data}

The targeted test engine is constantly being rebuilt to test new components and new control strategies. This implies that it is difficult to find measurement data from the same engine configuration. Most of the measurement data available is from the same layout as the data used in (Alegret et al., 2015). For layout number 1 the oxygen sensors were not properly calibrated and thus cannot be used for validating the oxygen levels at the manifolds. For the model parameterization 30 different stationary points are extracted from the measurement data and another 24 sta- 


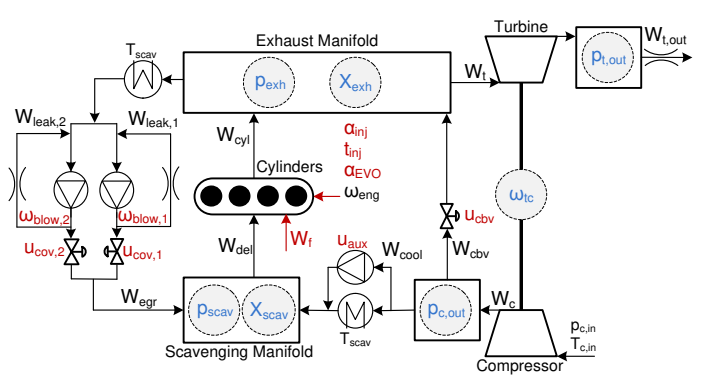

Figure 1. Engine model diagram with states (blue) and control inputs (red).

tionary points are saved for the validation.

Some more data is available from another layout of the engine and will be used for validation of the oxygen level in the scavenging manifold. However, in this layout, number 2, the turbocharger was changed and some sensors where removed. Moreover, there is much less data available, and only 18 stationary points could be extracted for the parameterization and the validation of the model.

\section{Modeling}

The complete MVEM model consists of thirteen states and nine control inputs. Figure 1 depicts a model diagram. The states are compressor outlet pressure, $p_{c, \text { out }}$, scavenging manifold pressure, $p_{s c a v}$, exhaust manifold pressure, $p_{\text {exh }}$, turbine outlet pressure, $p_{t, \text { out }}$ and turbocharger speed, $\omega_{t c}$. The chemical species mass fractions are states in the scavenging and the exhaust manifolds, $X_{\text {scav }}$ and $X_{\text {exh }}$. The species included in the model are oxygen, carbon dioxide, water and sulfur dioxide, $\mathrm{X}=\left[\mathrm{X}_{\mathrm{O}_{2}}, \mathrm{X}_{\mathrm{CO}_{2}}, \mathrm{X}_{\mathrm{H}_{2} \mathrm{O}}, \mathrm{XSO}_{2}\right]$. The dynamic equations are the same for each species so (6) and (7) correspond to eight single ODEs. The dynamic behavior of the modeled states is governed by the following differential equations

$$
\begin{aligned}
& \frac{d}{d t} \omega_{t c} \quad=\frac{P_{t}-P_{c}}{J_{t} \omega_{t c}} \\
& \frac{d}{d t} p_{c, \text { out }} \quad=\frac{R_{a} T_{c, \text { out }}}{V_{c, \text { out }}}\left(W_{c}-W_{\text {cool }}-W_{c b v}\right) \\
& \frac{d}{d t} p_{\text {scav }} \quad=\frac{R_{a} T_{\text {scav }}}{V_{\text {scav }}}\left(W_{\text {cool }}+W_{\text {egr }}-W_{\text {del }}\right) \\
& \frac{d}{d t} p_{e x h} \quad=\frac{R_{e} T_{e x h}}{V_{e x h}}\left(W_{c y l}-W_{e g r}-W_{t}+W_{c b v}\right) \\
& \frac{d}{d t} p_{t, \text { out }} \quad=\frac{R_{e} T_{t, \text { out }}}{V_{t, \text { out }}}\left(W_{t}-W_{t, \text { out }}\right) \\
& \frac{d}{d t} X_{\text {scav }} \quad=\frac{R_{a} T_{\text {scav }}}{p_{\text {scav }} V_{\text {scav }}}\left(X_{\text {exh }}-X_{\text {scav }}\right) W_{\text {egr }} \\
& +\frac{R_{a} T_{\text {scav }}}{p_{\text {scav }} V_{\text {scav }}}\left(X_{\text {amb }}-X_{\text {scav }}\right) W_{\text {cool }} \\
& \frac{d}{d t} X_{e x h} \quad=\frac{R_{e} T_{e x h}}{p_{e x h} V_{e x h}}\left(X_{c y l}-X_{e x h}\right) W_{c y l}
\end{aligned}
$$

The control inputs are EGR blower speeds, $\omega_{\text {blow }, 1}$ $\omega_{\text {blow }, 2}$, blower cut-out valves (COV) position, $u_{c o v, 1} u_{c o v, 2}$, fuel mass flow, $W_{f}$, fuel injection angle $\alpha_{i n j}$, fuel injection time, $t_{i n j}$, exhaust valve closing angle, $\alpha_{E V C}, \mathrm{CBV}$ position, $u_{c b v}$, and auxiliary blower operation $u_{a u x}$. Engine speed, $\omega_{e n g}$, and compressor inlet pressure and tempera- ture, $p_{c, \text { in }} T_{c, \text { in }}$, are considered known inputs to the model.

It is simple to reduce the model to seven states if we are only interested in tracking the oxygen level in the manifolds. Then in (6) and (7), the mass fraction only refers to oxygen, e.g. $X=X_{O_{2}}$. The model is built using different submodels interconnected. The submodels are mainly control volumes, e.g. scavenging manifold, and flow elements e.g. cylinder bypass valve, compressor, turbine, etc. Since the model is an extension of the one described in (Alegret et al., 2015), only the new or modified submodels are presented here.

\subsection{Compressor}

In order to properly simulate low loads, a compressor model capable of predicting mass flow and efficiency at low speeds is required. The chosen compressor mass flow model is the one developed in (Leufvén and Eriksson, 2014), which is capable of this extrapolation. In addition, the proposed model is capable of predicting mass flows down to pressure ratios below one and to zero compressor speed. The area where the compressor normally operates during low load is below the slowest measured speed line, which is depicted in Figures 2 and 3.

In the model, each compressor speed line is described by a super ellipse, which mathematically is written as

$$
\left(\frac{\bar{W}_{c}-\bar{W}_{Z S}}{\bar{W}_{C h}-\bar{W}_{Z S}}\right)^{C U R}+\left(\frac{\Pi_{c}-\Pi_{C h}}{\Pi_{Z S}-\Pi_{C h}}\right)^{C U R}=1
$$

where $\bar{W}_{Z S}, \bar{W}_{C h}, \Pi_{Z S}, \Pi_{C h}$ and $C U R$ are functions of compressor speed. More details about these functions and the model in general can be found in (Leufvén and Eriksson, 2014). Since (8) is invertible, it can be used to predict either pressure ratio given compressor speed and mass flow or mass flow given compressor speed and pressure ratio. The latter case is used in the proposed engine model.

The compressor efficiency is modeled using the ideas from (Martin et al., 2009) for the isentropic efficiency definition. The key for the model is to use the Euler's equation (Dixon and Hall, 2013) applied to the compressor velocity triangles. Using the simplifications from (Martin et al., 2009), the conclusion is that the actual enthalpy rise for a fixed compressor speed can be modeled as a linear function of the mass flow. This simplifies the number of parameters required and since it is based in the physical equations, makes the extrapolation to the low load area more reliable. The proposed compressor model requires 15 parameters for the mass flow submodel and 4 parameters for the efficiency submodel.

The mass flow model together with the efficiency model fitted to the two compressors used in this study is depicted in Figures 2 and 3. The absolute value of the relative errors for both compressors are shown in Table 1.

\subsection{Turbine}

The Turbine mass flow model is very similar to the one used in (Alegret et al., 2015). However, a modification in the model is required to describe the turbine speed depen- 


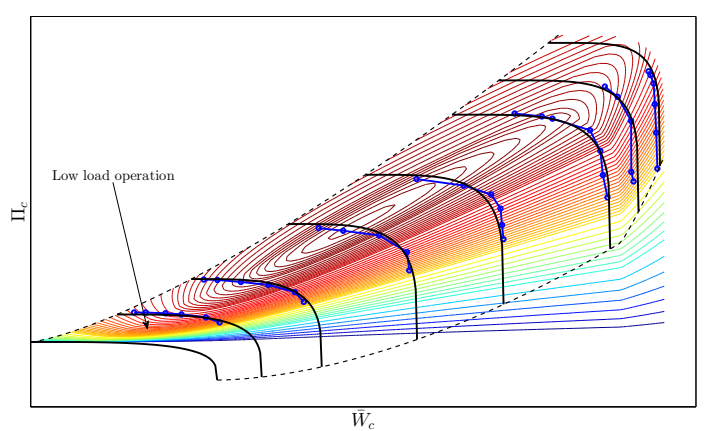

Figure 2. Compressor 1 model, in black, plotted together with the measured map points, in blue dots. The first speed line in the lower left corner represents the stand still characteristics of the compressor. The thinner level lines represent the modeled efficiency extrapolation down to unity pressure ratio.

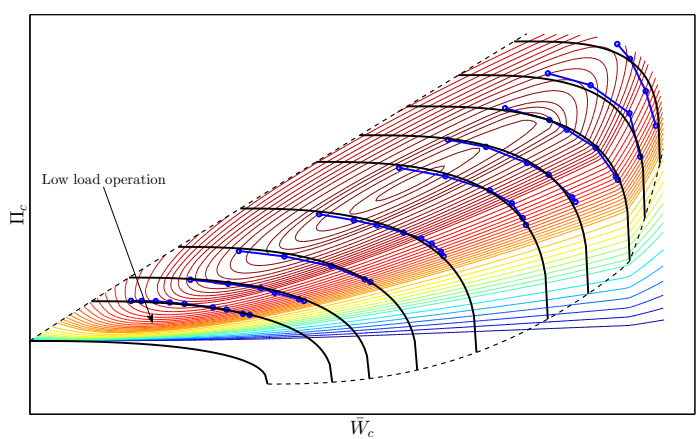

Figure 3. Compressor 2 model, in black, plotted together with the measured map points, in blue dots. The first speed line in the lower left corner represents the stand still characteristics of the compressor. The thinner level lines represent the modeled efficiency extrapolation down to unity pressure ratio.

dence observed for Turbine 2. The mass flow is described by the following function from (Eriksson and Nielsen, 2014)

$$
\bar{W}_{t}=C_{t} \sqrt{1-\left(\Pi_{t}+\Pi_{0}\right)^{k_{t}}}
$$

where $k_{t}, \Pi_{0}$ and $C_{t}$ are constant parameters to be estimated for Turbine 1. For Turbine $2, k_{t}$ and $C_{t}$ are also constants but $\Pi_{0}$ is modeled using a quadratic polynomial of corrected turbine speed to capture the different speed lines observed in Figure 5. The quadratic polynomial is defined as

$$
\Pi_{0}=c_{\Pi_{0}, 1} \bar{N}_{t}^{2}+c_{\Pi_{0}, 2} \bar{N}_{t}+c_{\Pi_{0}, 3}
$$

where $c_{\Pi_{0}, 1}, c_{\Pi_{0}, 2}$ and $c_{\Pi_{0}, 3}$ are model parameters. The two models fitted to the two turbines are shown on the left side of Figures 4 and 5.

Table 1. Absolute value of the relative errors (\%) for both compressors and turbines. Mean indicates the mean value of all errors while Max. is the maximum error computed.

\begin{tabular}{c|c|c|c|c|c|c|c|c|}
\cline { 2 - 9 } & \multicolumn{2}{c|}{ Compressor 1 } & \multicolumn{2}{c|}{ Compressor 2 } & \multicolumn{2}{c|}{ Turbine 1 } & \multicolumn{2}{c|}{ Turbine 2 } \\
\cline { 2 - 9 } & Mean & Max. & Mean & Max. & Mean & Max. & Mean & Max. \\
\hline $\bar{W}$ & 0.86 & 2.21 & 0.59 & 1.87 & 0.14 & 0.51 & 0.56 & 5.61 \\
\hline$\eta$ & 1.47 & 4.51 & 0.74 & 2.87 & 0.60 & 3.32 & 0.69 & 6.11 \\
\hline
\end{tabular}
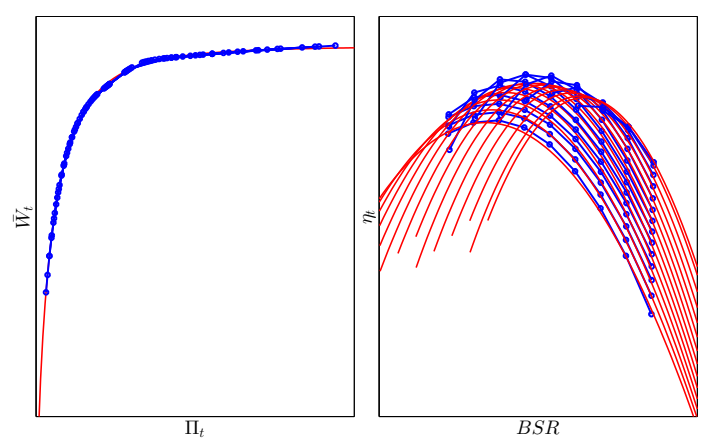

Figure 4. Left: turbine 1 mass flow model, in red, plotted together with the measured map points, in blue dots. Right: turbine 1 efficiency model, in red, plotted with the measured efficiency points, in blue dots.
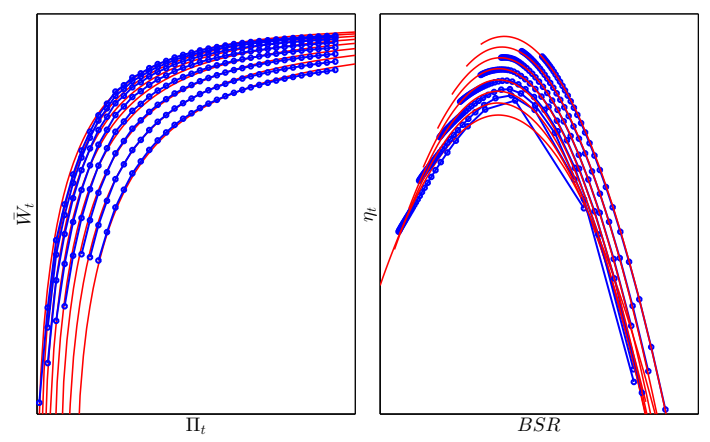

Figure 5. Left: turbine 2 mass flow model, in red, plotted together with the measured map points, in blue dots. Right: turbine 2 efficiency model, in red, plotted with the measured efficiency points, in blue dots.

The turbine efficiency is modeled using the Blade Speed Ratio (BSR), as in (Wahlström and Eriksson, 2011; Alegret et al., 2015). The relation between turbine efficiency and BSR is defined as

$$
\eta_{t}=c_{1} B S R^{2}+c_{2} B S R+c_{3}
$$

where $c_{1}, c_{2}$ and $c_{3}$ are also quadratic functions of turbine corrected speed, and each polynomial is defined as follows

$$
c_{X}=c_{X, 1} \bar{N}_{t}^{2}+c_{X, 2} \bar{N}_{t}+c_{X, 3}
$$

In total the turbine efficiency consists of nine parameters. The modeled and the measured efficiencies for both turbines are depicted in Figures 4 and 5. Furthermore, for both turbines, the absolute value of the relative errors are presented in Table 1.

\subsection{EGR Blowers}

The EGR blowers are modeled as in (Alegret et al., 2015). The only difference is that there are two equal blowers in parallel. The EGR flow is controlled with the blower speed control inputs, $\omega_{\text {blow, } 1}$ and $\omega_{\text {blow, } 2 \text {, and the cut-out }}$ valves are used to open or close the EGR flow. Depending on the engine running mode, there can be only one blower operating or both of them if more EGR flow is required. 


\subsection{Auxiliary Blower}

The pressure increase of an electric blower is often modeled as a quadratic function of the volumetric flow (Guan et al., 2014). Since the blower's pressure increase is available from the system states, the quadratic function is inverted to obtain the volumetric flow. Using the pressure and temperature at the inlet the mass flow provided by the blower is obtained

$$
W_{A u x}=\frac{p_{c, o u t}\left(c_{A u x, 1}+c_{A u x, 2} \sqrt{c_{A u x, 2}-\left(p_{s c a v}-p_{c, o u t}\right)}\right)}{R_{a} T_{c, \text { out }}}
$$

where $c_{A u x, 1}, c_{A u x, 2}$ and $c_{A u x, 3}$ are tuning parameters estimated with the blower technical specifications.

For the studied test engine, the auxiliary blower is installed in parallel with the air cooler after the compressor. This means that when operating it will pull air mass flow from the compressor outlet control volume to the scavenging manifold. When active, the pressure difference between these two control volumes will reverse. When the auxiliary blower is operated (since there is no restriction valve for reverse flow in the cooler), there is flow recirculation in the air cooler. This issue needs to be modeled in order to capture the measured system behavior. Thus, the flow from the compressor outlet to the scavenging manifold is then modeled using (13) and two incompressible flow restrictions from (Eriksson and Nielsen, 2014)

$$
W_{\text {cool }}= \begin{cases}W_{\text {Aux }}-A_{\text {cool }, r} \sqrt{\frac{p_{\text {scav }}\left(p_{\text {scav }}-p_{c, \text { out }}\right)}{T_{\text {scav }}}} & \text { if } u_{\text {aux }}=1 \\ A_{\text {cool }} \sqrt{\frac{p_{c, \text { out }}\left(p_{c, \text { out }}-p_{\text {scav }}\right)}{T_{c, \text { out }}}} & \text { if } u_{\text {aux }}=0\end{cases}
$$

where $A_{\text {cool }}$ represents the flow restriction when the blower is inactive and $A_{c o o l, r}$ models the magnitude of the recirculation when the blower is running. Both are parameters to be estimated.

\subsection{Exhaust Back Pressure}

An incompressible flow restriction together with a control volume is used to model the back pressure for the turbine, $p_{t, \text { out }}$. The pressure dynamics are described by (5). And the exhaust flow $W_{t, \text { out }}$ is modeled using the standard incompressible flow restriction from (Eriksson and Nielsen, 2014) where the restriction area is a tuning parameter.

\subsection{Combustion Species and Thermodynamic Parameters}

The species mass fraction out of the cylinders, $X_{c y l}$, is calculated using the stoichiometric combustion equation and the air and fuel flows entering the cylinders. Without including the nitrogen explicitly, the combustion equation can be written

$$
\mathrm{CH}_{y} \mathrm{~S}_{z}+(1+y / 4+z) \mathrm{O}_{2}=\mathrm{CO}_{2}+(y / 2) \mathrm{H}_{2} \mathrm{O}+z \mathrm{SO}_{2}
$$

where $y$ is the hydrogen to carbon ratio and $z$ the amount of sulfur in the fuel, which are known parameters. In the case of the reduced model with only oxygen mass fraction, $X_{c y l}$ can be computed as in (Alegret et al., 2015; Wahlström and Eriksson, 2011).

Furthermore, the species vector is used to compute the thermodynamic parameters, $R, c_{p}$ and $\gamma$ of the working gas. This is done for each different gas composition and computed together with the gas temperature using the Nasa polynomials that can be found in (Goodwin et al., 2014).

\section{Parameterization Procedure}

The parameterization is done in similar steps as it is described in (Alegret et al., 2015). First the following submodels are parameterized alone: compressor, turbine, ERG blowers and Aux blower. These submodel parameters are kept fixed in the following parameterization steps.

\subsection{Complete stationary parameterization}

The path to follow would be to estimate the different flow restrictions of the model independently and then do a complete parameterization of the whole model together. However, this is not possible since there is no mass flow measurement available. Hence, the next step in the parameterization is to use the complete model to get the best set of parameters that predict the measured states.

The method followed here differs from the one used previously in (Alegret et al., 2015) where the derivatives of the states are used in the parameterization. Here instead the whole model is simulated at each stationary point and the simulated stationary states are used to compute the relative errors, $e_{r e l}$. Finally, a least-squares problem is formulated with the following objective function

$$
V_{\text {stat }}(\theta)=\frac{1}{N S} \sum_{i=1}^{S} \sum_{n=1}^{N}\left(e_{r e l}^{i}[n]\right)^{2}
$$

where $S$ is the number of different measured signals used to compute the relative errors, in the general case those signals are: $\left[p_{\text {scav }}, p_{\text {exh }}, p_{c, \text { out }}, p_{t, \text { out }}, \omega_{t c}, T_{\text {exh }}, P_{\text {eng }}, W_{\text {egr }}\right]$. $N$ is the number of stationary points used. The vector $\theta$ represents the parameters to be estimated, which in this case are all the static parameters, except of the fixed parameters of the submodels stated in the beginning of Section 4. The stationary simulations are done using a Matlab/Simulink implementation of the model. To reduce the computational time required, the Matlab parallel computing toolbox is used to run simultaneous simulations. Furthermore, a check on the state derivatives is done in order to stop the simulation once the stationary levels are reached.

Different estimation steps are done to ensure that the solver does not get lost with too many parameters. The parameterization is started without EGR, CBV or low load stationary points which are progressively included in the successive steps. Also, the results from each parameterization step are used as initial guess for the following one. Finally, a complete parameterization with all stationary points available is done. 
Table 2. Absolute value of the model relative errors (\%) for both engine layouts. Low load is with Aux. blower active, Mid load is below $70 \%$ and High load is above $70 \%$

\begin{tabular}{c|c|c|c|c|c|c|c|c|}
\cline { 2 - 9 } & \multicolumn{8}{|c|}{ Engine Layout 1 } \\
\cline { 2 - 10 } & $p_{\text {scav }}$ & $p_{\text {exh }}$ & $p_{c, \text { out }}$ & $p_{t, \text { out }}$ & $\omega_{t c}$ & $T_{\text {exh }}$ & $P_{\text {eng, } i}$ & $W_{\text {egr }}$ \\
\hline Low Load & 2.39 & 2.62 & 5.13 & 2.07 & 2.80 & 0.80 & 0.44 & 11.44 \\
\hline Mid Load & 3.51 & 3.36 & 6.86 & 3.37 & 1.90 & 0.78 & 0.36 & 9.84 \\
\hline High Load & 4.74 & 6.92 & 2.05 & 5.29 & 2.58 & 1.28 & 2.52 & 5.55 \\
\hline & \multicolumn{7}{|c|}{ Engine Layout 2} \\
\cline { 2 - 10 } & $p_{\text {scav }}$ & $p_{\text {exh }}$ & $p_{c, \text { out }}$ & $p_{t, \text { out }}$ & $\omega_{t c}$ & $T_{\text {exh }}$ & $X_{O_{2}}$ & - \\
\hline Low Load & 4.75 & 7.73 & 4.38 & 0.17 & 6.32 & 1.74 & 1.93 & - \\
\hline Mid Load & 3.75 & 3.08 & 4.78 & 0.18 & 2.26 & 1.59 & 1.03 & - \\
\hline High Load & 7.69 & 10.48 & 4.42 & 0.48 & 2.35 & 3.48 & 0.89 & - \\
\hline
\end{tabular}

\subsection{Dynamic estimation}

Fixing the estimated parameters at the previous steps, the dynamic parameters, $J_{t}, V_{\text {scav }}, V_{e x h}, V_{c, \text { out }}, V_{t, \text { out }}$ and $\tau_{c o v}$, are tuned using the same procedure as in (Alegret et al., 2015). In this case, 17 different step responses are used, including tree load steps with the auxiliary blowers active.

\section{Model Validation}

Table 2 presents the absolute value of the relative errors separated for different load ranges. For Layout 1, the 24 validation stationary points are used. For Layout 2 , since there is few data available, the errors are computed with the same stationary points used in the estimation. For both Layouts, the higher pressure errors are mostly in the high load case, where also the exhaust temperature and indicated engine power errors are higher. This indicates that the model and in particular the Seiliger cycle could be improved in this area. One reason for this could be that at high load is where the engine protection controls limit the maximum pressure in the cylinders, and this might not be totally captured in the model. On the other hand, for the mid and low load ranges the errors are in general of similar magnitude. Note that for the Layout 2, the engine power and the EGR mass flow measurements are not available.

Figure 6 shows the simulation results of Layout 1 compared to the measurements of a dataset not used in the estimation. This simulation has a low load phase where the auxiliary blower is enabled, where it can be seen that the system behavior is captured by the model. In particular the measured pressure and turbocharger speed values are matched by the model. More discrepancy is observed in the modeled exhaust temperature during the transients.

For Layout 2, the simulation results are presented in Figure 7. There is also a low load operation that the model is capable to capture. In this case the turbocharger speed prediction is worse than for the Layout 1 which in turn affects the stationary levels of the pressures. Nevertheless, it is important to mention that it has been parameterized with few data. Oxygen mass fraction validation could not be done due to unreliable measurements for Layout 1 and in the previous investigations from (Alegret et al., 2015).

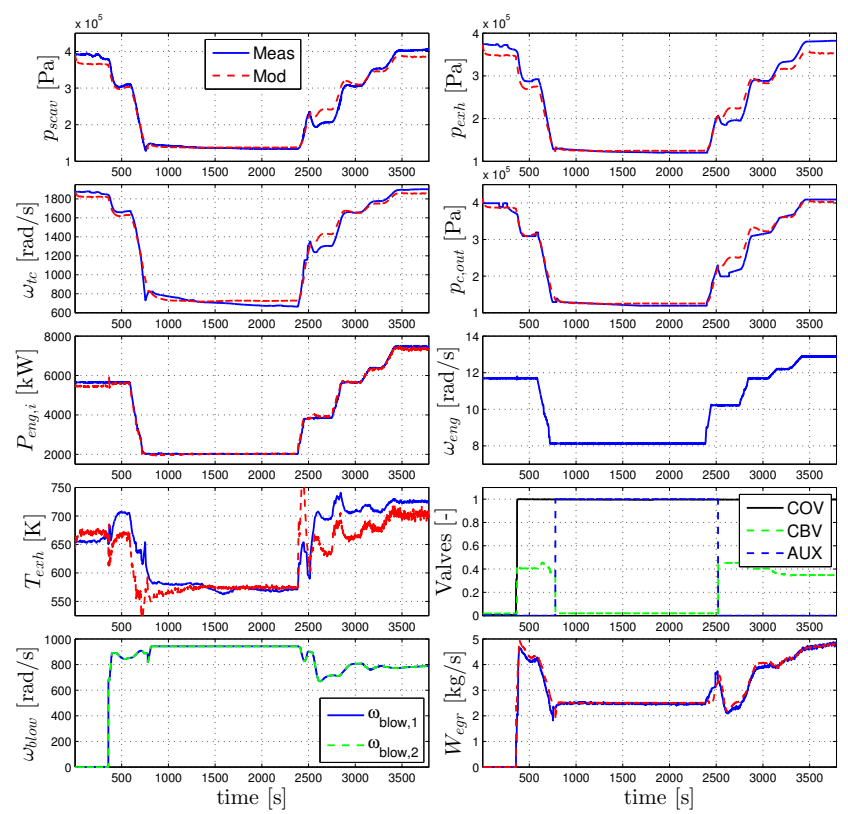

Figure 6. Model simulation vs measurements for Engine Layout 1.

Therefore, the most relevant result from Layout 2 is that the scavenging oxygen level is captured as it can be seen in Table 2 and in Figure 7.

\section{Conclusions}

An MVEM for a marine two-stroke diesel engine capable of simulating low loads is proposed and validated. A parameterization method to overcome the lack of mass flow measurements is also proposed. The main characteristic is that it uses a Simulink model to integrate the modeled states with stationary inputs and is used to compute the residuals. Two different layouts of the same engine but with different turbochargers are investigated, the results show a good agreement between simulation results and measurements with some room for improvement at high loads. The oxygen prediction capabilities are also validated for the second engine layout since the oxygen measurements are reliable.

\section{Acknowledgment}

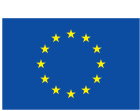

This project has received funding from the European Union's Horizon 2020 research and innovation programme under grant agreement No 634135.

MAN Diesel \& Turbo and in particular Guillem Alegret are also acknowledged for their support and suggestions about the modeled test engine.

\section{A Nomenclature}

\section{References}

Guillem Alegret, Xavier Llamas, Morten Vejlgaard-Laursen, and Lars Eriksson. Modeling of a large marine two-stroke diesel engine with cylinder bypass valve and EGR system. 


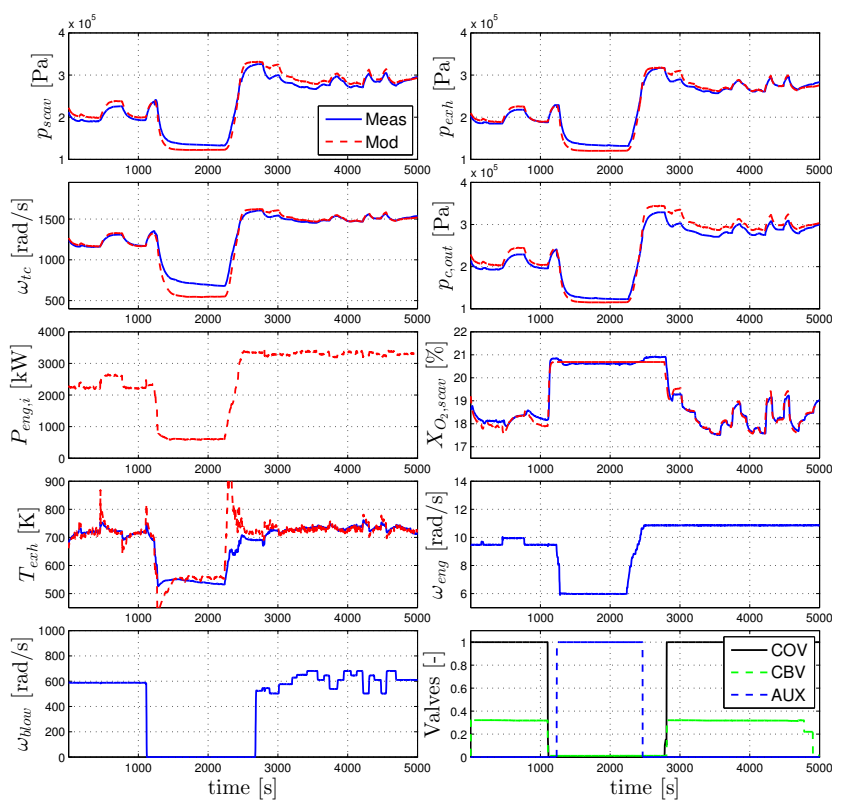

Figure 7. Model simulation vs measurements of for Engine Layout 2.

IFAC-PapersOnLine, 48(16):273 - 278, 2015. ISSN 24058963. doi:http://dx.doi.org/10.1016/j.ifacol.2015.10.292. 10th IFAC Conference on Manoeuvring and Control of Marine Craft MCMC.

M. Blanke and J. A. Anderson. On modelling large two stroke diesel engines: new results from identification. IFAC Proceedings Series, pages 2015-2020, 1985.

Sydney Lawrence Dixon and Cesare A Hall. Fluid Mechanics and Thermodynamics of Turbomachinery. ButterworthHeinemann, 7th edition, 2013.

Lars Eriksson and Lars Nielsen. Modeling and Control of Engines and Drivelines. John Wiley \& Sons, 2014.

David G. Goodwin, Harry K. Moffat, and Raymond L. Speth. Cantera: An object-oriented software toolkit for chemical kinetics, thermodynamics, and transport processes. http: // www . cantera.org, 2014. Version 2.1.2.

C. Guan, G. Theotokatos, P. Zhou, and H. Chen. Computational investigation of a large containership propulsion engine operation at slow steaming conditions. Applied Energy, 130: 370-383, 2014. doi:10.1016/j.apenergy.2014.05.063.

Table 3. Subscripts

\begin{tabular}{l|l||l|l} 
cov & cut-out valve & inj & injection \\
blow & blower & measured \\
mod & modeled \\
scav & scavenging manifold & cool & cooler \\
cyl & cylinder & $t$ & turbine \\
del & delivered & eng & engine \\
$e$ & exhaust gas & $a$ & air \\
tc & turbocharger & $x$, in & inlet of $\mathrm{x}$ \\
aux & auxiliary blower & $x$, out & outlet of $\mathrm{x}$ \\
exh & exhaust manifold & egr & EGR gas
\end{tabular}

Table 4. List of symbols

\begin{tabular}{c|l|c}
$A$ & Area & {$\left[\mathrm{m}^{2}\right]$} \\
$c_{p}$ & Specific heat at constant pressure & {$[\mathrm{J} /(\mathrm{kgK})]$} \\
$J$ & Inertia & {$\left[\mathrm{kg} \mathrm{m}^{2}\right]$} \\
$W$ & Mass flow & {$[\mathrm{kg} / \mathrm{s}]$} \\
$\bar{W}$ & Corrected mass flow & {$[\mathrm{kg} / \mathrm{s}]$} \\
$p$ & Pressure & {$[\mathrm{Pa}]$} \\
$P$ & Power & {$[\mathrm{kW}]$} \\
$R$ & Gas constant & {$[\mathrm{J} /(\mathrm{kgK})]$} \\
$T$ & Temperature & {$[\mathrm{K}]$} \\
$V$ & Volume & {$\left[\mathrm{m}^{3}\right]$} \\
$X$ & Mass fraction & {$[-]$} \\
$\alpha$ & angle & {$[\mathrm{rad}]$} \\
$\gamma$ & Specific heat capacity ratio & {$[-]$} \\
$\eta$ & Efficiency & {$[-]$} \\
$\Pi$ & Pressure ratio & {$[-]$} \\
$\bar{N}$ & Corrected rotational speed & {$[\mathrm{rpm}]$} \\
$\omega$ & Rotational speed & {$[\mathrm{rad} / \mathrm{s}]$}
\end{tabular}

Jakob Mahler Hansen, Claes-Göran Zander, Nikolai Pedersen, Mogens Blanke, and Morten Vejlgaard-Laursen. Modelling for control of exhaust gas recirculation on large diesel engines. Proceedings of the 9th IFAC conference on Control Applications in Marine Systems, 2013.

International Maritime Organization. MARPOL: Annex VI and NTC 2008 with Guidelines for Implementation. IMO, 2013. ISBN 978-92-801-15604.

Oskar Leufvén and Lars Eriksson. Measurement, analysis and modeling of centrifugal compressor flow for low pressure ratios. Int. J. Engine Res., pages 1-16, December 2014. doi: $10.1177 / 1468087414562456$.

Guillaume Martin, Vincent Talon, Pascal Higelin, Alain Charlet, and Christian Caillol. Implementing turbomachinery physics into data map-based turbocharger models. SAE Int. J. of Engines, 2(1):211-229, April 2009. doi:10.4271/2009-01-0310.

M.J. Nieuwstadt, I.V. Kolmanovsky, P.E. Moraal, A. Stefanopoulou, and M. Jankovic. EGR-VGT control schemes: experimental comparison for a high-speed diesel engine. IEEE Control Systems Mag., 20(3):63-79, 2000.

G. Theotokatos. On the cycle mean value modelling of a large two-stroke marine diesel engine. Proceedings of the Institution of Mechanical Engineers, Part M: Journal of engineering for the maritime environment, 224(3):193-206, 2010. ISSN 1475-0902.

J. Wahlström and L. Eriksson. Modelling diesel engines with a variable-geometry turbocharger and exhaust gas recirculation by optimization of model parameters for capturing non-linear system dynamics. Proceedings of the Institution of Mechanical Engineers, Part D: Journal of Automobile Engineering, 225:960-986, 2011. 